\title{
Increasingly complex DNA assemblies
}

\author{
Prompted by advances in the programmability of DNA nanostructures and their hybridization, the complexity of \\ nanomaterial lattices guided by DNA continues to increase.
}

T: he role of DNA as a structural guiding motif and linker for molecular assembly and crystal engineering ${ }^{1,2}$ is now established in various branches of materials science. We now have a fairly comprehensive understanding of how the size, shape and morphology of DNA-grafted nanoparticles and the sequence, length, flexibility and density of DNA linkers contribute to the interaction energy between each nanoparticles ${ }^{1,2}$. Moreover, DNA scaffolds composed of multiple helices or self-folded DNA origami tiles that bind or enclose nanoparticles have been developed $^{3}$. These design rules and building blocks enable one to exert control over the position, orientation and periodicity of the individual nanoparticles and their arrangement into ordered lattices, which can have uses in photonic, plasmonic or catalytic applications, among others ${ }^{4-6}$.

We have previously highlighted the versatility of DNA linkers for the rational design of nanoparticle crystals with structural complexity ${ }^{7}$. In this issue, we bring together several research papers and a Comment to showcase how the prescribed complexity of DNA-guided assembly is being harnessed for the creation of a broad range of designer material systems, from single crystals at interfaces to three-dimensional lattices of proteins.

Controlling colloidal crystallization at an interface is challenging since both interparticle and particle-substrate interactions need to be considered and modulated. In a Letter, Robert Macfarlane and colleagues explore the programmability of DNA hybridization between DNA-grafted gold nanoparticles and substrate-bound strands to individually modulate the crystal-substrate, crystal-fluid and substrate-fluid interfacial energies, and assemble single-crystalline gold nanoparticle Winterbottom constructions. These structures represent the thermodynamically stable shape of a crystal grown at an interface. The authors show that crystal size, orientation and anisotropy can be adjusted using the design handles of nanoparticle concentration, solution ionic strength, and DNA grafting density on the particles and surfaces. As noted in the accompanying News \& Views from Chang Liu and Qian Chen, these single-crystalline constructions could be assembled periodically on graphene

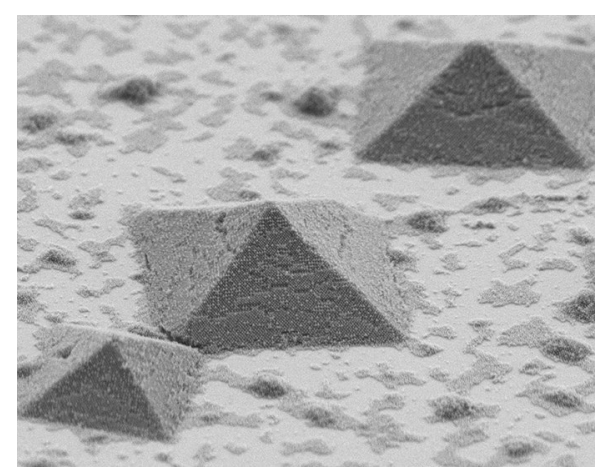

Winterbottom constructions of DNA-gold nanoparticles. Courtesy of Robert J. Macfarlane.

for bandgap structure engineering, which may be useful for electronic devices.

The programmability of DNA structures and their hybridization is described by Oleg Gang and colleagues in an Article reporting polyhedral DNA origami frames that encapsulate distinct nanomaterials, from metal nanoparticles to enzymes, and organize them into three-dimensional crystals. Lattice geometry and symmetry are defined by the shape, valence and coordination of the DNA scaffolds, whose vertex contains six DNA strands capable of hybridization with those attached to the vertex of another frame. The assembly process is thus decoupled from the nature of the encapsulated nanomaterial, being solely governed by the DNA scaffolds. In a related News \& Views, Veikko Linko and Mauri Kostiainen highlight that this design paradigm is a major step into the realm of intelligent nanofabrication, which could pave the way towards more compelling optoelectronics devices. Indeed, the technological potential of this approach is supported by the demonstrations of optically and biologically active crystals with tuneable quantum dot emission and enzymatic reaction cascades included in the Article.

While DNA interactions are crucial for DNA-nanoparticle constructions, the functionalization of nanoparticles with DNA is an equally important step and advances in this chemistry have also significantly contributed to the increased complexity of DNA-nanoparticle lattices ${ }^{8,9}$. An Article by Chunhai Fan, Ben Feringa and colleagues describes a method to impart gold nanoparticles with valence bond analogues made from single-stranded DNA. These strands contain polyadenine domains that irreversibly attached to the gold particle and non-polyadenine domains whose sequence, length and order can be programmed to modulate the arrangement of the DNAnanoparticle into low-coordination colloidal assemblies with particular composition, sizes and geometries. The versatility of DNA pairing interactions allows the authors to dynamically reconfigure the composition and structure of the colloidal assemblies and even explore it for the implementation of molecular logic gates.

The studies mentioned above are only a few examples of recent achievements of DNA nanotechnology for the design of ordered nanomaterials systems. In a Comment, Hao Yan and colleagues discuss many other cases, how all of these have led to an ever-growing number of structurally intricate assemblies and crystals, and some of the challenges that lie ahead. As argued by these researchers, a significant step forward would be the development of a general assembly method that would allow binding any nanomaterial (inorganic to biological) into functional, responsive lattices with desired lattice geometries and symmetries that could be used in emergent technological applications.

Overall, DNA-guided assembly of nanomaterials continues to be a very active field of research. Despite the increasing number of reports describing more and more complex DNA-nanomaterial assemblies, we are still eager to know what else can be constructed through DNA-mediated assembly.

Published online: 24 June 2020

https://doi.org/10.1038/s41563-020-0727-3

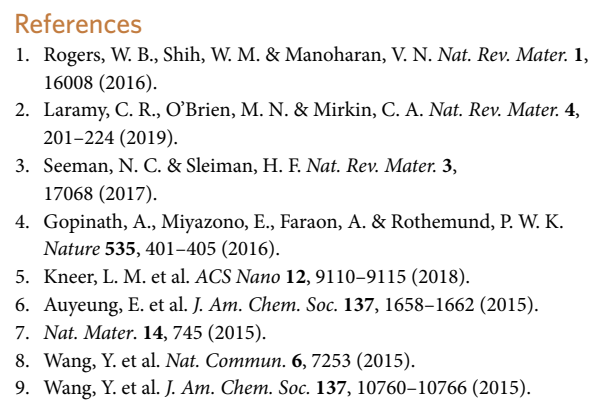

2. Laramy, C. R., O’Brien, M. N. \& Mirkin, C. A. Nat. Rev. Mater. 4, 201-224 (2019).

3. Seeman, N. C. \& Sleiman, H. F. Nat. Rev. Mater. 3, 17068 (2017).

4. Gopinath, A., Miyazono, E., Faraon, A. \& Rothemund, P. W. K. Nature 535, 401-405 (2016).

5. Kneer, L. M. et al. ACS Nano 12, 9110-9115 (2018).

6. Auyeung, E. et al. J. Am. Chem. Soc. 137, 1658-1662 (2015).

7. Nat. Mater. 14, 745 (2015).

8. Wang, Y. et al. Nat. Commun. 6, 7253 (2015).

9. Wang, Y. et al. J. Am. Chem. Soc. 137, 10760-10766 (2015). 